Y. Miyata

Nagoya Math. J.

Vol. 54 (1974), 53-59

\title{
ON THE MODULE STRUCTURE OF THE RING OF ALL INTEGERS OF A p-ADIC NUMBER FIELD
}

\author{
YOSHIMASA MIYATA
}

Let $k$ be a $p$-adic number field and $o$ be the ring of all integers of $k$. Let $K / k$ be a cyclic ramified extension of prime degree $p$ with Galois group $G$. Then the ring $\subseteq$ of all integers of $K$ is $v[G]$-module. The purpose of this paper is to give a necessary and sufficient condition for $o[G]$-module $\subseteq$ to be indecomposable.

In $\S \S 1-2$, we shall prepare some lemmas. In $\S 3$, we shall obtain the necessary and sufficient condition (Theorem 1).

1. In this section, we shall construct an arithmetical sequence of rational integers and study its properties. We begin with defining sequences $a_{1}^{i}, a_{2}^{i}, \cdots, a_{p-1}^{i}$ for $1 \leqq i \leqq p-1$. Sequences $a_{j}^{i}$ are defined inductively by:

$$
\begin{aligned}
& a_{1}^{1}=1, a_{2}^{1}=2, \cdots, a_{p-1}^{1}=p-1 \\
& a_{1}^{2}=0, a_{2}^{2}=a_{1}^{1}, a_{3}^{2}=a_{1}^{1}+a_{2}^{1}, \cdots, a_{p-1}^{2}=a_{1}^{1}+a_{2}^{1}+\cdots+a_{p-2}^{1}, \cdots, \\
& a_{1}^{i}=0, \cdots, a_{i-1}^{i}=0, a_{i}^{i}=a_{i-1}^{i-1}, a_{i+1}^{i}=a_{i-1}^{i-1}+a_{i}^{i-1}, \cdots, \\
& a_{p-1}^{i}=a_{i-1}^{i-1}+a_{i}^{i-1}+\cdots+a_{p-2}^{i-1}, \cdots
\end{aligned}
$$

We evaluate $a_{j}^{i}$.

LEMMA 1.

$$
a_{j}^{i}=\frac{\{j-(i-1)\}\{j-(i-2)\} \cdots j}{i !} \quad \text { for } 1 \leqq i \leqq j \leqq p-1 .
$$

Proof. We use induction on $i$. The result is trivial for $i=1$. Let $i>1$, and suppose the result holds for $a_{j}^{i^{\prime}}$ where $1 \leqq i^{\prime} \leqq i-1$ and $i^{\prime} \leqq$ $j \leqq p-1$. Then we have 


$$
\begin{aligned}
a_{j}^{i}= & a_{i-1}^{i-1}+\cdots+a_{j-1}^{i-1}=\frac{1}{(i-1) !}[\{(i-1)-(i-1-1)\} \cdots(i-1) \\
& +\cdots+\{(j-1)-(i-1-1)\} \cdots(j-1)] \\
= & \frac{1}{(i-1) !}\left[\sum_{a=1}^{(j-1)-(i-2)}\left\{\prod_{b=0}^{i-2}(a+b)\right\}\right] .
\end{aligned}
$$

From the formula $\sum_{a=1}^{j}\left\{\prod_{b=0}^{i-1}(a+b)\right\}=1 /(i+1) \prod_{b=0}^{i}(j+b)$ ([5]), we obtain

$$
a_{j}^{i}=\frac{1}{(i-1) !} \frac{1}{i} \prod_{b=0}^{i-1}\{j-(i-1)+b\}
$$

This proves the lemma.

We may observe that the above proof also yields the following lemma :

LEMMA 2. $\left(a_{i}^{i}+a_{i+1}^{i}+\cdots+a_{p-1}^{i}\right) / p$ is an integer of the field of $p$-adic numbers for $1 \leqq i<p-1$.

Now, let $\theta$ be a primitive $p$-th root of 1 .

LEMMA 3. $\theta^{j}-1=a_{j}^{1}(\theta-1)+\cdots+a_{j}^{j}(\theta-1)^{j}$ for $1 \leqq j \leqq p-1$.

Proof. We use induction on $j$. The result is clear for $j=1$. Assume it holds $j \leqq j_{0}-1$. We shall prove that it holds for $j=j_{0}$ Then

$$
\begin{aligned}
\left(\theta^{j_{0}}-1\right) /(\theta-1) & =a_{j_{0}}^{1}+\left(\theta^{j_{0}-1}-1\right)+\cdots+(\theta-1) \\
& =a_{j_{0}}^{1}+(\theta-1)\left(\sum_{h=1}^{j_{0}-1} a_{h}^{1}\right)+\cdots+(\theta-1)^{j_{0}-1} a_{j_{0}-1}^{j_{0}-1} .
\end{aligned}
$$

Hence by the definition of $a_{j_{0}}^{i}$ we have

$$
\theta^{j_{0}}-1=a_{j_{0}}^{1}(\theta-1)+a_{j_{0}}^{2}(\theta-1)^{2}+\cdots+a_{j_{0}}^{j_{0}}(\theta-1)^{j^{0}} .
$$

2. Let $K / k$ be a cyclic ramified extension of prime degree $p$. In this section, we shall evaluate valuations of some elements of $K$. Let $\Pi$ and $D$ be a prime element of $K$ and the different of $K / k$. Let $e$ denote the absolute ramification index of $k$. Let $\mathfrak{D}=\subseteq \Pi^{n}$. We can write $n$ in the form $n=p m+l$ with $0 \leqq l<p$. Let $g$ be a generator of the Galois group $G$ of $K / k$ and $c$ be the first ramification number of $K / k$. By the definition of $c$, we have $g(\Pi)=\Pi+u \Pi^{c+1}$, where $u$ is a unit of $K$. As is well known,

$$
m \leqq e, \text { and } p m+l=(p-1)(c+1) .
$$


Now, take any integer $\alpha$ of $K$. For $\alpha$ we define a sequence of $p-1$ integers $\alpha_{0}, \alpha_{1}, \cdots, \alpha_{p-2}$ inductively by:

$$
\alpha_{0}=\alpha, \alpha_{1}=g\left(\alpha_{0}\right)-\alpha_{0}, \cdots, \quad \text { and } \quad \alpha_{p_{-2}}=g\left(\alpha_{p-3}\right)-\alpha_{p-3} .
$$

We shall evaluate the valuation of $\alpha_{j}$. Let $\nu_{k}$ denote the valuation of $K\left(\nu_{k}(\Pi)=1\right)$.

LEMMA 4. Let $a=\nu_{k}(\alpha)$. Then $\nu_{k}\left(\alpha_{j}\right) \geqq \min (a+j c, p m+1$, pe).

Proof. $a$ can be written in the form $a=p q+r$ with $0 \leqq r<p$. Let $\left(\Pi^{p m+1}, \Pi^{p e}\right)$ be the ideal generated by $\Pi^{p m+1}$ and $\Pi^{p e}$. Since $e \geqq m$ and $p m+1<p(c+1)$, we have

$$
\begin{aligned}
g\left(\Pi^{a}\right) & =g\left(\Pi^{p q+r}\right)=\left(\Pi+u \Pi^{c+1}\right)^{p q+r} \\
& \equiv \Pi^{p q+r}\left(1+u \Pi^{c}\right)^{r} \quad \bmod .\left(\Pi^{p m+1}, \Pi^{p e}\right) .
\end{aligned}
$$

Put $\left(1+u \Pi^{c}\right)^{r}=1+r u^{\prime} \Pi^{c}$. Hence

$$
g\left(\Pi^{a}\right) \equiv \Pi^{a}\left(1+r u^{\prime} \Pi^{c}\right) \quad \bmod .\left(\Pi^{p m+1}, \Pi^{p e}\right) .
$$

Now let $j=1$. We can write $\alpha=U \Pi^{a}$, where $U$ is a unit of $\supseteqq$. Then, by (2), we have

$$
\begin{aligned}
\alpha_{1} & =g(\alpha)-\alpha=g(U) g\left(\Pi^{a}\right)-U \Pi^{a} \\
& \equiv \Pi^{a}\left\{g(U)\left(1+r u^{\prime} \Pi^{c}\right)-U\right\} \quad \bmod .\left(\Pi^{p m+1}, \Pi^{p e}\right) .
\end{aligned}
$$

We have $g(U)=U+v \Pi^{c+1}$, where $v$ is an integer of $K$. Hence

$$
\alpha_{1} \equiv \Pi^{a+c}\left\{r u^{\prime} U+v \Pi+r u^{\prime} \Pi^{c+1}\right) \quad \bmod .\left(\Pi^{p m+1}, \Pi^{p e}\right) .
$$

Therefore, we obtain the inequality for $j=1$, and simulately, we obtain it also for $j>1$.

Now, let $k_{0}$ be the field $k(\theta)$ and $K_{0}$ the field $K(\theta)$. Let $e_{0}$ denote the degree of $k_{0} / k$. Since the extension $k_{0} / k$ is tamely ramified, we have $k_{0} \cap K=k$. Therefore there exists a unique element $g_{0}$ of the Galois group $G_{0}$ of $K_{0} / k_{0}$ such that for any element $\alpha$ of $K$

$$
g_{0}(\alpha)=g(\alpha)([3]) \text {. }
$$

Let $\mathfrak{D}_{0}$ be the ring of all integers of $K_{0}$ and define the element $E$ of the group ring $k_{0}[G]$ by

$$
E=\sum_{j=1}^{p} \theta^{j-1} g_{0}^{p-j}
$$


In the following we shall obtain a congruence for $E \alpha$ (where $\alpha$ is an integer of $K$ as before). Keeping the same notations as in preceding Lemma 4 , define $\alpha_{j}^{i}$ by $\alpha_{j}^{i}=g_{0}^{i-1}\left(\alpha_{j}\right)$. We obtain the following lemma.

Lemma 5. Let $E, g_{0}, \alpha, \alpha_{j}^{i}$ be as above. Let $p^{\prime}$ be $p^{\prime}=\max (p-2,1)$. Then

$$
E \alpha \equiv\left(\sum_{j=1}^{p} g_{0}^{j-1}\right) \alpha+\sum_{i=1}^{p^{\prime}}(\theta-1)^{i} \alpha_{p-i-1} \quad \bmod . \mathfrak{D}_{0} p
$$

Proof. We immediately obtain the result for $p=2$, and so suppose hereafter $p \geqq 3$. Then we have $p^{\prime}=p-2$ and

$$
\left(E-\sum g_{0}^{j-1}\right) \alpha \equiv \sum_{j=1}^{p-2}\left(\theta^{j}-1\right)\left(\sum_{h=1}^{p-j-1} \alpha_{1}^{h}\right) \quad \bmod . \mathfrak{D}_{0} p .
$$

Then, by Lemma 3, we have

$$
\left(E-\sum g_{0}^{j-1}\right) \alpha \equiv \sum(\theta-1)^{i}\left\{\sum_{j=i}^{p-2} \alpha_{j}^{i}\left(\sum_{h=1}^{p-j-1} \alpha_{1}^{h}\right)\right\} \quad \bmod . \mathfrak{D}_{0} p .
$$

First we investigate the case $i=1$. By the definition of $\alpha_{j}^{2}$,

$$
\sum_{j=1}^{p-2} a_{j}^{1}\left(\sum_{h=1}^{p-j-1} \alpha_{1}^{h}\right)=\sum_{h=1}^{p-2}\left(\sum_{j=1}^{p-h-1} a_{j}^{1}\right) \alpha_{1}^{h}=\sum_{h=1}^{p-2} \alpha_{p-h}^{2} \alpha_{1}^{h} .
$$

Put $p-h=j$. Then

$$
\begin{aligned}
& =\sum_{j=2}^{p-1} a_{j}^{2} \alpha_{1}^{p-j} \\
& =\sum_{j=2}^{p-2} a_{j}^{2}\left(\alpha_{1}^{p-j}-\alpha_{1}^{1}\right)+\left(\sum_{j=2}^{p-1} a_{j}^{2}\right) \alpha_{1}^{1} \\
& =\sum_{j=2}^{p-2} a_{j}^{2}\left(\sum_{h=1}^{p-j-1} \alpha_{2}^{h}\right)+\left(\sum_{j=2}^{p-1} a_{j}^{2}\right) \alpha_{1}^{1}
\end{aligned}
$$

By Lemma 2, $\left(\sum a_{j}^{2}\right) \alpha_{1}^{1} \in \mathfrak{D}_{0} p$. Therefore

$$
\sum_{j=1}^{p-2} a_{j}^{1}\left(\sum_{h=1}^{p-j-1} \alpha_{1}^{h}\right) \equiv \sum_{j=2}^{p-2} a_{j}^{2}\left(\sum_{h=1}^{p-j-1} \alpha_{2}^{h}\right) \quad \bmod . \mathfrak{D}_{0} p .
$$

Repeating this process, we obtain

$$
\sum_{j=1}^{p-2} a_{j}^{1}\left(\sum_{h=1}^{p-j-1} \alpha_{1}^{h}\right) \equiv \sum_{j=i}^{p-2} a_{j}^{i}\left(\sum_{h=1}^{p-j-1} \alpha_{i}^{h}\right) \quad \bmod . \mathfrak{D}_{0} p .
$$

Then, 


$$
\begin{aligned}
\sum a_{j}^{1}\left(\sum \alpha_{1}^{h}\right) & \equiv \sum a_{j}^{i}\left(\sum \alpha_{i}^{h}\right) \\
& \equiv \sum_{h=1}^{p-i-1}\left(\sum_{j=i}^{p-h-1} a_{j}^{i}\right) \alpha_{i}^{h} \\
& \equiv \sum_{h=1}^{p-i-1} a_{p-h}^{i+1} \alpha_{i}^{h}
\end{aligned}
$$

Put $p-h=j$.

$$
\begin{aligned}
& \equiv \sum_{j=i+1}^{p-1} a_{j}^{i+1} \alpha_{i}^{p-j} \\
& \equiv \sum_{j=i+1}^{p-2} a_{j}^{i+1}\left(\alpha_{i}^{p-j}-\alpha_{i}^{1}\right)+\left(\sum_{j=i+1}^{p-1} a_{j}^{i+1}\right) a_{i}^{1} \quad \bmod \mathfrak{D}_{0} p .
\end{aligned}
$$

Using Lemma 2 again, we obtain $\left(\sum a_{j}^{i+1}\right) \alpha_{i}^{1} \in \mathfrak{D}_{0} p$. Hence

$$
\sum a_{j}^{1}\left(\sum \alpha_{1}^{h}\right) \equiv \sum_{j=i+1}^{p-2} a_{j}^{i+1}\left(\sum_{h=1}^{p-j-1} \alpha_{j+1}^{h}\right) \quad \bmod . \mathfrak{S}_{0} p .
$$

Therefore we have

$$
(\theta-1)\left\{\sum a_{j}^{1}\left(\sum a_{1}^{h}\right)\right\} \equiv(\theta-1) a_{p-2}^{p-2} a_{p-2}^{1} \quad \bmod . \mathfrak{D}_{0} p .
$$

Applying the same arguments, for $i \geqq 2$ we have

$$
(\theta-1)^{i}\left\{\sum_{j=i}^{p-2} a_{j}^{i}\left(\sum_{n=1}^{p-j-1} \alpha_{1}^{h}\right)\right\} \equiv(\theta-1)^{i} a_{p-2}^{p-2} a_{p-i-1}^{1} \quad \bmod . \mathfrak{D}_{0} p .
$$

From $a_{p-2}^{p-2}=1$, for $1 \leqq i \leqq p-2$ we have

$$
(\theta-1)^{i}\left\{\sum \alpha_{j}^{i}\left(\sum \alpha_{1}^{h}\right)\right\} \equiv(\theta-1)^{i} \alpha_{p-i-1}^{1} \quad \bmod . \mathfrak{D}_{0} p .
$$

The lemma is proved.

3. We shall use the same notations as in the previous section. Let $\pi$ be a prime element of $k$, and write $\mathfrak{D}=\subseteq \Pi^{p m+l}$ as before. At first, we observe that there is a prime element $\Pi^{\prime}$ such that $\left(\sum_{j=1}^{p} g^{j-1}\right) \Pi^{\prime p-t-1}$ $\in \mathfrak{D} \pi^{m}$ and $\notin \mathfrak{D} \pi^{m+1}([2])$. Therefore we may have

$$
\nu_{k}\left\{\left(\sum g^{j-1}\right) \Pi^{p-l-1}\right\}=p m .
$$

Next, from the result of E. Maus ([4] (3.19)), we have the following lemma :

LEMMA 6. The first ramification number $c_{0}$ of the extension $K_{0} / k_{0}$ is $e_{0} c$.

Finally, we obtain the next theorem which is the aim of this paper. 
THEOREM 1. Let $K / k$ be a cyclic ramified extension of prime degree $p$, and $e$ be the absolute ramification index of $k$. Let $\mathfrak{D}, \mathfrak{o}, G$, and $m$ be as stated before. Then $\mathfrak{S}$ is an indecomposable $\mathrm{o}[G]-$ module if and only if $m<e$.

This is obviously equivalent with the following theorem that we shall prove in the following:

THEOREM 2. Let $K / k, \mathfrak{O}, \mathrm{D}, G, e, m$. be as in Theorem 1. Then $\mathfrak{D}$ is decomposable if and only if $m=e$.

Proof. At first we suppose $m=e$. As is well known, $\left(\sum_{j=1}^{p} g^{j-1}\right) \mathfrak{O}$

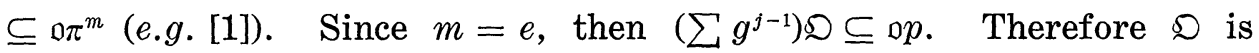
decomposable.

Conversely, we suppose that $\mathfrak{D}$ is decomposable. Then there are o[G]-submodules $\mathfrak{D}_{1}$ and $\mathfrak{D}_{2}$ of $\mathfrak{D}$ such that

$$
\mathfrak{D}=\mathfrak{D}_{1} \oplus \mathfrak{D}_{2}
$$

As the $k[G]$-module $K(=k \lesssim)$ is isomorphic to $k[G]$, we have

$$
k \bigcirc_{1} \cong \sum_{\left\{x_{j}\right\}}\left\{\frac{\sum_{i=1}^{p-1} \chi_{j}\left(g^{-i}\right) g^{i}}{p}\right\} k \circlearrowleft
$$

where $\left\{\chi_{j}\right\}$ are irreducible $k$-character of $G$. Then we see that the element $\sum_{\left\{\chi_{j}\right\}}\left\{\sum \chi_{j}\left(g^{-i}\right) g^{i}\right\} / p$ is an $\mathfrak{o}[G]$-endomorphism of $\mathfrak{D}$. Hence

$$
\sum_{\left\{\chi_{j}\right\}}\left\{\sum \chi_{j}\left(g^{-i}\right) g^{i}\right\} \subseteq \subseteq Ð p .
$$

Now $\chi_{j}$ is a direct sum of irreducible $k_{0}$-character $\chi_{j r}$ :

$$
\chi_{j}=\chi_{j 1}+\cdots+\chi_{j r_{j}} .
$$

Then $\sum \chi_{j}\left(g^{-i}\right) g^{i} / p$ is a sum of central idempotents $\sum \chi_{j r}\left(g^{-i}\right) g^{i} / p$. Let $E_{j r}=\sum_{i=0}^{p-1} \chi_{j r}\left(g^{-i}\right) g^{i}$. Then we have

$$
\sum_{\left\{\chi_{j}\right\}}\left(\sum \chi_{j}\left(g^{-i}\right) g^{i}\right)=\sum_{j, r} E_{j r} .
$$

Let $s$ denote the number of the set $E_{j r}$ (i.e. $s=\sum_{j} r_{j}$ ). By Lemma 5 and (3), we have $\left(\sum E_{j r}\right) \Pi^{p-l-1} \equiv s\left(\sum g^{j-1}\right) \Pi^{p-l-1}+\sum_{\left\{\theta_{j r}\right\}}\left\{\sum_{i=1}^{p^{\prime}}\left(\theta_{j r}-1\right)^{i}\right.$ $\left.\alpha_{p-i-1}\right\} \bmod . \mathfrak{D}_{0} p$, where $\left\{\theta_{j r}\right\}$ are $\chi_{j r}\left(g^{-1}\right)$. From (5) and (6),

$$
s\left(\sum g^{j-1}\right) \Pi^{p-l-1}+\sum_{\left\{\theta_{j r}\right]}\left(\sum\left(\theta_{j r}-1\right)^{i} \alpha_{p-i-1}^{1}\right) \equiv 0 \quad \bmod . \mathfrak{Ð}_{0} p .
$$


Furthermore, it follows from Lemma 4 that

$$
\nu_{k}\left\{\alpha_{p-i-1}^{1}\right\} \geqq \min (p-l-1+c(p-i-1), p m+1, p e) .
$$

Let $\nu_{k_{0}}$ denote the valuation of $K_{0}$ (i.e. $\nu_{k_{0}}(I)=e_{0}$ ). Then

$$
\nu_{k_{0}}\left\{(\theta-1)^{i} \alpha_{p-i-1}^{1}\right\} \geqq \min \left(N, p m e_{0}+1, p e e_{0}\right),
$$

where

$$
N=\{p-l-1+c(p-i-1)\} e_{0}+\frac{p e e_{0}}{p-1} i
$$

As $(c+1)(p-1)=p m+l$, we have $(p-1) c+p-l-1=p m$. Here we note that

$$
\begin{aligned}
N & =\{(p-l-1)+(p-1) c\} e_{0}+\left(\frac{p e e_{0}}{p-1}-c e_{0}\right) i \\
& =p m e_{0}+\left(\frac{p e e_{0}}{p-1}-c e_{0}\right) i
\end{aligned}
$$

First, we consider the case that $p e e_{0} /(p-1)=c e_{0}$. Then, from (1), we obtain $m=e$. Next, we consider the case that $p e e_{0} /(p-1)>c e_{0}$. Then we have $N>p m e_{0}$. Therefore, by (7) and (8),

$$
\nu_{k_{0}}\left\{s\left(\sum g^{j-1}\right) \Pi^{p-l-1}\right\} \geqq p e e_{0} .
$$

As $\mathfrak{S}_{2} \neq\{0\}, 1 \leqq s<p$. Then $s$ is a unit of $\mathfrak{D}_{0}$. It follows from (4) and (9) that $p m e_{0} \geqq p e e_{0}$. This implies $m=e$, and the proof of the theorem is completed.

\section{REFERENCES}

[1] E. Artin and J. Tate, Class Field Theory, Benjamin, New York, 1967.

[2] S. Amano, Eisenstein equations of degree $p$ in a p-adic field, J. Fac. Sci. Univ. Tokyo vol. 18, No 1 (1971), 1-21.

[ 3 ] N. Bourbaki, Èléments de Mathématique Algèbre Chap. 4 et 5, Hermann, Paris, 1959.

[ 4 ] E. Maus, Arithmetisch disjunkte Körper, J. reine angew. Math. 226 (1967), 184203.

[ 5 ] Sūgaku Jiten (Mathematical dictonary), Iwanami, Tokyo, 1968.

Faculty of Education, Shizuoka University 